\title{
AN INTERVAL ESTIMATOR FOR CHLORINE MONITORING IN DRINKING WATER DISTRIBUTION SYSTEMS UNDER UNCERTAIN SYSTEM DYNAMICS, INPUTS AND CHLORINE CONCENTRATION MEASUREMENT ERRORS
}

\author{
RAFAŁ ŁANGOWSKI $^{a, *}$ MIETEK A. BRDYS ${ }^{a, b}$ \\ ${ }^{a}$ Department of Control Systems Engineering \\ Gdańsk University of Technology, G. Narutowicza 11/12, 80-233 Gdańsk, Poland \\ e-mail: rafal.langowskilepg.gda.pl \\ ${ }^{b}$ School of Electronic, Electrical and Computer Engineering, College of Engineering and Physical Sciences \\ University of Birmingham, Edgbaston, Birmingham B15 2TT, UK
}

\begin{abstract}
The design of an interval observer for estimation of unmeasured state variables with application to drinking water distribution systems is described. In particular, the design process of such an observer is considered for estimation of the water quality described by the concentration of free chlorine. The interval observer is derived to produce the robust interval bounds on the estimated water quality state variables. The stability and robustness of the interval observer are investigated under uncertainty in system dynamics, inputs, initial conditions and measurement errors. The bounds on the estimated variables are generated by solving two systems of first-order ordinary differential equations. For that reason, despite a large scale of the systems, the numerical efficiency is sufficient for the on-line monitoring of the water quality. Finally, in order to validate the performance of the observer, it is applied to the model of a real water distribution network.
\end{abstract}

Keywords: observers, bounding methods, modelling dynamics, water quality.

\section{Introduction}

Drinking water distribution systems (DWDSs) are part of critical infrastructure systems (EU Cost Action IC0806-IntelliCIS, 2008), in which reliable, high performance and secure operation is very important, due to the need for ensuring safe and efficient functioning of the society.

The main objective for the DWDS is to meet demand on water of required quality for all domestic and industrial consumers (Brdys and Ulanicki, 1994). As this goal is complex, suitable monitoring and control algorithms operating on-line need to be applied in order to achieve it. Moreover, two aspects must be taken into account during control and monitoring in the DWDS: quantity and water quality. It was shown by Brdys et al. (1995) that a strong, unilateral relation between quantity and quality exists. Hydraulics of water has an impact on the water quality; therefore, the necessity of an integrated approach

* Corresponding author to control the DWDS appears. A very important aspect of efficient operation of the DWDS is gathering on-line information. In the DWDS two types of monitoring systems can be distinguished: the water quantity and water quality. In this paper only the water quality monitoring system is considered. The water quality can be determined by several quality factors and a disinfectant concentration is one of the most popular ones. Several disinfectants are used in practice but the chlorine is the leading one. The water quality monitoring system exploits the water quality measurements in order to gather knowledge about the state of water quality. In typical DWDSs water quality sensors are located at the network nodes. The water quality may be measured in laboratories or by using on-line sensors. Bacteriology measurements (e.g., the number of coli bacteria) are typical laboratory measurements in the DWDS and obviously, they cannot be utilised on-line. At present, primarily the free chlorine concentration may be measured on-line. Hence in this paper the water quality is determined by its concentration. 
Unfortunately, the chlorine sensor placement at all of the DWDS nodes is impossible. It is due to, e.g., the high costs of hard sensors and the possibility of their their installation. Therefore, the missing information about the state needs to be recovered by employing their estimation based on other measurements and, e.g., the mathematical model of the process.

Typically, the DWDS is composed of pumps, valves, pipes, nodes, tanks and reservoirs. The pumps and valves are used to control hydraulic quantities that are the water flow rates and pressures. Since the chlorine reacts with organic and non-organic matter in water, the chlorine concentration decreases in time (Males et al., 1988; Rossman et al., 1994). On the one hand, if the chlorine concentration is too low, pathogenic bacteria can grow in water and this can even lead to the bacteriological instability. On the other hand, a strong chlorination performed at the water treatment stations and within the network by booster stations can recover safe water, but can also lead to an increase in the concentration of harmful disinfection by-products (DBPs) (Boccelli et al., 2003; Arminski et al., 2013). Therefore, the proper values of the chlorine concentration should be guaranteed by a suitable activity of all the water treatment stations in the DWDS. In this paper, modelling of the DBPs is not considered. Hence, the DWDS water quality modelling takes into account changes in the chlorine concentration at the nodes, tanks and along the pipes. Moreover, it is assumed that reservoirs are the sources of treat water (the water quality from reservoirs is constant).

During formulation of the decay models of the chlorine concentration, it is commonly assumed that a hydraulic solution of the DWDS (the values of flow rates at the pipes, etc.) is known and it is constant over the specified time interval called the hydraulic step. The models of chlorine decay during water transfer through the pipes can be found in the works of Males et al. (1988), Rossman et al. (1994), Park and Kuo (1996), Al-Omari and Chaudhry (2001). During water transfer throughout the network, the water may be stored in tanks. The water retention time in a tank can be long and unfavourably influence the water quality in the DWDS. The long water retention time in the tanks may lead to a decrease in the chlorine concentration, regrowth of the pathogenic bacteria in water and the appearance of the DBPs. Therefore, modelling the chlorine decay in the tanks is necessary and it can be found in the work of Mau et al. (1995) or Clark et al. (1996). In this paper it is assumed that the tank is a continuous stirred reactor (CSR) and the quality model has been shown by Clark et al. (1996). In the DWDS there are controlled and uncontrolled quality nodes (Boulos et al., 2004). When modelling water quality it is assumed that the instantaneous and complete mixing of chlorine at both types of nodes proceeds. Therefore, the chlorine concentration out of the node at all outflows is the same and it is equal to the chlorine concentration at the node. The quality controlled nodes are the nodes with chlorine booster stations. Chlorine is directly dosed into the controlled quality nodes in order to maintain the required chlorine concentration, which is prescribed by the quality controller (Propato et al., 2001).

Hence the DWDS water quality model is composed of the system of algebraic equations that describe the changes of chlorine concentration at the nodes, the system of ordinary differential equations that describe the chlorine decay in the tanks and the system of partial differential equations that describe the distribution in the chlorine concentrations along the network pipes. There are several methods which can be used in order to solve this model (Rossman and Boulos, 1996). One of them is the finite-difference method based on discretization of partial differential equations in the time and space domains (Mitchel and Griffiths, 1980). Another option is discretization only in space. An outline of this methodology was given by Łangowski and Brdys (2007). In turn, a comprehensive approach is described in this paper.

The process also includes modelling the uncertainty. There are several approaches to uncertainty modelling; however, a set-membership approach is an attractive option (Brdys and Chen, 1995; Amairi, 2016; Jauberthie et al., 2016). In this approach the uncertainty is described by means of an additive bounded error where only the bounds are known. The bounded models of uncertainty need less a-priori information about the system and they are less demanding than the probabilistic models. The optimisation based on the set-membership algorithms for set bounded estimation of the quantity, integrated quantity and quality in the DWDS was presented by Brdys and Chen (1995), Duzinkiewicz (2006) as well as Brdys and Chen (1996), respectively. Unfortunately, due to the necessity of solving highly nonlinear and nonconvex optimisation problems, it is time-consuming and, thus, not always applicable to on-line estimation. Hence a new interval estimation method for quality monitoring in the DWDS is proposed in this paper. The method utilises an observer known as an interval observer.

In the literature various approaches to design interval observers can be found. For example, an interesting survey of the structures of interval observers was presented by Efimov and Raïssi (2016). In this paper a structure which was applied to the wastewater treatment process (Gouzé et al., 2000; Hadj-Sadok and Gouzé, 2001; Alcaraz-González et al., 2005; Rapaport and Dochain, 2005) is used and further developed. The first approach to set bounded estimation with the interval observer for an example DWDS was presented by Łangowski and Brdys (2006). Next, the structure of the observer was further developed and presented by Łangowski and Brdys (2007). The structures of interval 
observer, utilising only the direct state measurements were shown in these papers. Further research was focused on deriving the proof of stability and robustness of the designed interval observer and it was generally outlined by Brdys and Łangowski (2008). In this paper a comprehensive approach also containing a new structure of interval observer is presented. Moreover, the proposed observer is applied to water quality estimation in a real DWDS and its performance is validated by simulation. The detailed research on modelling and estimation with the interval observer can be found in the work of Łangowski (2015).

\section{DWDS water quality model}

The model of advective chlorine transport with the first-order decay for the $l$-th pipe can be described by (Males et al., 1988; Rossman et al., 1994; Park and Kuo, 1996; Al-Omari and Chaudhry, 2001)

$$
\partial_{t} c_{l}(z, t)+v_{l}(z, t) \partial_{z} c_{l}(z, t)=\alpha_{l} c_{l}(z, t),
$$

with the initial and boundary conditions

$$
c_{l}(z, 0)=c_{l, 0, t}(z), \quad c_{l}(0, t)=c_{l, 0, z}(t),
$$

where $t$ is the time instant; $z$ is the distance along the $l$-th pipe, $z=0$ and $z=L_{l}$ denote the beginning and the end of the pipe, respectively; $\partial_{(\cdot)}$ denotes the partial derivative with respect to $(\cdot) ; c_{l}(z, t)$ signifies the chlorine concentration within the $l$-th pipe at the distance $z$ at the instant $t ; v_{l}(z, t)$ denotes the linear water flow velocity in the $l$-th pipe; $\alpha_{l}$ stands for the bulk reaction rate coefficient of the $l$-th pipe.

Remark 1. Notice that the linear water flow velocity and flow rate are the same at a given pipe, i.e., $v_{l}(z, t)=$ $v_{l}(t)$. This is because of the assumption of the water incompressibility and that pipes are of a pressure type.

The water quality model in the CSR can be described as (Clark et al., 1996)

$$
\begin{aligned}
\mathrm{d}_{t}\left(V_{f, h} c_{f, h}\right)= & S_{1}-c_{f, h}(t) S_{2}+\beta_{h} V_{f, h}(t) c_{f, h}(t) \\
& \mathrm{d}_{t} V_{f, h}(t)=S_{3}-S_{2}
\end{aligned}
$$

with the initial conditions

$$
V_{f, h}(0)=V_{f, h, 0}, \quad c_{f, h}(0)=c_{f, h, 0} .
$$

Moreover, the following condition must be taken into account for the storage tank with a separated water filling in and drawing cycles:

$$
S_{2} S_{3}=0
$$

where

$$
S_{1}=\sum_{l \in \mathrm{IN} f, h(t)} q_{l}(t) c_{l}\left(L_{l}, t\right),
$$

$$
\begin{gathered}
S_{2}=\sum_{l \in \mathrm{OUT} f, h(t)} q_{l}(t), \\
S_{3}=\sum_{l \in \mathrm{INf}, h(t)} q_{l}(t) .
\end{gathered}
$$

Here $d_{t}$ stands for the derivative with respect to $t$; IN $f, h(t)$ is the set of pipes supplying water to the tank $h$ at the instant $t$; OUT $f, h(t)$ denotes the set of pipes draining water from tank $h$ at instant $t ; c_{f, h}(t)$ is the chlorine concentration in tank $h$ and at instant $t ; L_{l}$ signifies the length of pipe $l ; c_{l}\left(L_{l}, t\right)$ stands for the chlorine concentration at the end of the $l$-th pipe at instant $t ; V_{f, h}(t)$ signifies the volume of tank $h$ at instant $t ; q_{l}(t)$ denotes the corresponding water flow rates; $\beta_{h}$ is the tank reaction rate coefficient of the $h$-th tank.

Combining (3) and (2) the changes in the chlorine concentration in the tank can be described as

$$
\mathrm{d}_{t} c_{f, h}(t)=\left(-\frac{S_{3}}{V_{f, h}(t)}+\beta_{h}\right) c_{f, h}(t)+\frac{S_{1}}{V_{f, h}(t)},
$$

and for the storage tank with separated water filling in and drawing cycles during the drawing phase:

$$
d_{t} c_{f, h}(t)=\beta_{h} c_{f, h}(t)
$$

During the filling phase, the equation describing the chlorine concentration dynamics in the tank is the same as (5).

From the constituent mass balance law, the model of the chlorine decay at the $n$-th quality controlled node is (Boulos et al., 2004):

$$
S_{4}+S_{5}-c_{\mathrm{out}, n}(t)\left[S_{6}+S_{7}\right]+c_{\mathrm{doz}, n}(t)=0,
$$

where

$$
\begin{gathered}
S_{4}=\sum_{l \in \mathbb{I N} n(t)} q_{l}(t) c_{l}\left(L_{l}, t\right), \\
S_{5}=\sum_{l \in \operatorname{EIN} n(t)} q_{l}(t) c_{l}\left(L_{l}, t\right), \\
S_{6}=\sum_{l \in \operatorname{OUT} n(t)} q_{l}(t), \\
S_{7}=\sum_{l \in \operatorname{EOUT} n(t)} q_{l}(t) .
\end{gathered}
$$

$\operatorname{IN} n(t), \operatorname{EIN} n(t)$ are the sets of pipes supplying water and supplying water from the external sources to node $n$ at instant $t$, respectively; OUT $n(t), \operatorname{EOUT} n(t)$ denote the sets of pipes draining water into the network and draining water from node $n$ at instant $t$, respectively; $c_{\text {out }, n}(t)$ signifies the chlorine concentration at node $n$ at instant $t ; c_{\mathrm{doz}, n}(t)$ is the mass of chlorine injected to node $n$ at instant $t$. 
The chlorine concentration $c_{\text {out }, n}(t)$ can thus be written as

$$
c_{\mathrm{out}, n}(t)=\frac{c_{\mathrm{doz}, n}(t)}{S_{6}+S_{7}}+\frac{S_{4}+S_{5}}{S_{6}+S_{7}} .
$$

Denoting by $c_{i n, n}(t)$ the first term on the right-hand side of (8) yields

$$
c_{\mathrm{doz}, n}(t)=c_{\mathrm{in}, n}(t)\left[S_{6}+S_{7}\right] .
$$

Hence combining (9) and (8) gives

$$
c_{\mathrm{out}, n}(t)=c_{\mathrm{in}, n}(t)+\frac{S_{4}+S_{5}}{S_{6}+S_{7}} .
$$

We have the mass balance law

$$
S_{8}+S_{9}=S_{6}+S_{7},
$$

where

$$
S_{8}=\sum_{l \in \operatorname{IN} n(t)} q_{l}(t), \quad S_{9}=\sum_{l \in \operatorname{EIN} n(t)} q_{l}(t) .
$$

Hence, from (11) and (10) the following holds (Propato et al., 2001; Boulos et al., 2004):

$$
c_{\mathrm{out}, n}(t)=c_{\mathrm{in}, n}(t)+\frac{S_{4}+S_{5}}{S_{8}+S_{9}} .
$$

By contrast, water quality at the uncontrolled nodes is only dependent on the chlorine concentrations at the pipes supplying water into these nodes. Clearly, if there is no chlorine injection at node $n$, the first term on the right-hand side of (12) disappears (Rossman and Boulos, 1996; Boulos et al., 2004):

$$
c_{\text {out }, n}(t)=\frac{S_{4}+S_{5}}{S_{8}+S_{9}} .
$$

Note that the chlorine concentration in tank $h$ and at uncontrolled node $n$ at instant $t$ directly depends on the chlorine concentrations $c_{l}\left(L_{l}, t\right)$, where $l$ is from the set $\operatorname{IN} f, h(t), \operatorname{IN} n(t)$ and $\operatorname{EIN} n(t)$, respectively. In other words, these chlorine concentrations depend on the chlorine concentrations at the ends of the pipes supplying water into the tank and the node at instant $t$. Notice that the magnitudes and directions of water flow rates determine the water quality model structure. Since the flow directions and their magnitudes can change over the time horizon considered, the water quality model structure can change as well. In this paper they are assumed constant over a specified time interval called the hydraulic step, $T_{H}$ (Brdys and Ulanicki, 1994).
2.1. DWDS water quality model for estimation. In order to solve the DWDS water quality model a method of Mitchel and Griffiths (1980) can be used to discretize (1) in time and space domains. Clearly, in order to approximate (1), time $t$ and distance $z$ are discretized. Assume that for the $l$-th pipe $t=k \Delta t_{l}$, where $\Delta t_{l}$ stands for a time period and $z=m \Delta l_{l}, \Delta l_{l}$ signifies the length of the pipe segment. Writing $c_{l}(m, k)=c_{l}\left(m \Delta l_{l}, k \Delta t_{l}\right)$, the particular components of (1) can be approximated as follows (Chen, 1997):

$$
\begin{aligned}
&\left.\partial_{t} c_{l}(z, t)\right|_{z, t} \cong \frac{c_{l}(m, k+1)-c_{l}(m, k)}{\Delta t_{l}}, \\
&\left.\partial_{z} c_{l}(z, t)\right|_{z, t} \cong \frac{c_{l}(m, k)-c_{l}(m-1, k)}{\Delta l_{l}}, \\
& c_{l}(z, t)=\frac{c_{l}(m, k+1)+c_{l}(m, k)}{2} .
\end{aligned}
$$

By contrast, in the work of Łangowski and Brdys (2007) (11) was discretized only in the space domain. Clearly, if the time period $\Delta t_{l}$ approaches zero then the right-hand side of (14) approaches the derivative:

$$
\begin{aligned}
\left.\partial_{t} c_{l}(z, t)\right|_{z, t} \cong \frac{c_{l}(m, k+1)-c_{l}(m, k)}{\Delta t_{l}} \\
\underset{\Delta t_{l} \rightarrow 0}{\longrightarrow} \mathrm{d}_{t} c_{l}(m, t) .
\end{aligned}
$$

Moreover, (15) and (16) may be written as

$$
\begin{aligned}
\left.\partial_{z} c_{l}(z, t)\right|_{z, t} & \cong \frac{c_{l}(m, t)-c_{l}(m-1, t)}{\Delta l_{l}}, \\
c_{l}(z, t) & =c_{l}(m, t) .
\end{aligned}
$$

Therefore, (1) can be approximated as

$$
\begin{array}{r}
d_{t} c_{l}(m, t)+v_{l}(m, t) \frac{c_{l}(m, t)-c_{l}(m-1, t)}{\Delta l_{l}} \\
=\alpha_{l} c_{l}(m, t)
\end{array}
$$

with the initial conditions $c_{l}(m, 0)=c_{l, 0, t}(m)$.

Hence (20) describes the chlorine concentration over time at the pipe point located at the distance $m \Delta l_{l}$ from the pipe origin. Moreover, the discretized equation illustrates a virtual pipe partitioning into segments. The pipe partitioning method was described by Rossman et al. (1993) or Łangowski and Brdys (2007). During the partitioning procedure the travelling time of the chlorine concentration within each pipe over a particular $T_{H}$ is calculated. The minimum value of this time is the so-called quality step and is denoted by $T_{Q}$. Notice that two extreme cases can occur: (a) very short pipes with high linear water flow velocities and (b) very long pipes with slow linear water flow velocities (Rossman et al., 1993). Hence $T_{Q}$ and $N_{l}$ can yield a very small value in case (a) and a large number in case (b). Both cases 
may lead to a significant increase in the computational time. Therefore, a minimum quality step $T_{Q, \min }$ and a maximal number of segments $N_{l, \text { max }}$ are introduced. In consequence, a certain loss of solution accuracy appears, especially for the pipes with a short (case (a)) and a long (case (b)) travelling time.

Notice that the linear water flow velocity and flow rate at each segment within a given pipe are the same (see Remark 1). Moreover, the linear water flow velocity and flow rate at a given pipe can be assumed constant over $T_{H}$. A value of $T_{H}$ depends on the demand rate of change and is typically between 0.5 and 2 [h] (Brdys and Ulanicki, 1994). Clearly, the number of constant flow rate intervals over a time horizon $T$ equals $J=T / T_{H}$. The partitioning procedure is repeated for each $T_{H}$. Hence the number of segments within a single pipe may differ in each $T_{H}$. Therefore, the water quality model has a time varying structure. Hence, (5)-(6), (12)-(13) and (20) describe a time continuous lumped water quality model for estimation.

The chlorine concentrations at the network nodes and tanks are seen as most important and they are viewed as the DWDS quality outputs. The chlorine concentrations at the network nodes can be calculated by applying (12) and (13). Clearly, the forms of the equations depend on the node structures. Hence, the chlorine concentration at a given node requires the inflow rates as well as the chlorine concentrations at a given node. As was mentioned, the chlorine concentration at a given node depends on the chlorine concentrations at the ends of the pipes supplying water to the given node. Therefore, these chlorine concentrations can be determined by applying (20), whereas the chlorine concentrations in the tanks can be calculated by using (5) and (6). Moreover, the chlorine concentrations in the tanks as well as the chlorine concentrations at the pipe ends are the quality state variables and they are denoted by $\mathbf{x}(t)$.

Notice that the state variables representing the chlorine concentrations in the tanks are directly transferred to the system output. A similar situation occurs at a node supplied by only one pipe. Due to different travelling times through the pipes in the DWDS, pipe partitioning into segments is applied. The number of elementary segments within each pipe can be different and the chlorine concentration dynamics for each segment is defined by (20). This introduces new state variables into the water quality model. Hence the quality state $\mathbf{x}(t)$ is composed of the chlorine concentrations at all the pipe segment ends as well as in the tanks. Notice that the structure of vector $\mathbf{x}(t)$ is constant but only over each $T_{H}$. The state equations for the pipe segment ends are derived from (20). Let us denote by $x_{r}(t)$ the chlorine concentration at the end of the pipe segment $r$. Then from
(20) it follows that (Łangowski and Brdys, 2007)

$$
\mathrm{d}_{t} x_{r}(t)=\left(-\frac{v_{r}(t)}{\Delta l_{r}}+\alpha_{l}\right) x_{r}(t)+\frac{v_{r}(t)}{\Delta l_{r}} c_{e, l, r}(t),
$$

where $v_{r}(t)$ denotes the linear water flow velocity along the segment $r ; \Delta l_{r}$ is the length of segment $r ; c_{e, l, r}(t)$ signifies the chlorine concentration at the beginning of the $r$-th segment.

Four cases for the segment quality input $c_{e, l, r}(t)$ can be distinguished:

(i) $c_{e, l, r}(t)=x_{r-1}(t)$,

(ii) $c_{e, l, r}(t)=c_{s r}(t)$,

(iii) $c_{e, l, r}(t)=x_{h}(t)$,

(iv) $c_{e, l, r}(t)=c_{\text {out }, n}(t)$,

where $x_{r-1}(t), c_{s r}(t), x_{h}(t), c_{\mathrm{out}, n}(t)$ are the chlorine concentrations at instant $t$ at the end of segment $r-1$, the external water source, tank $h$ and node $n$ calculated from (12) or 13, respectively.

As the state equations for the tanks are derived from (5) and (6), we get

$$
\begin{aligned}
& \mathrm{d}_{t} x_{h}(t)=\left(-\frac{S_{3}}{V_{f, h}(t)}+\beta_{h}\right) x_{h}(t) \\
&+\frac{\sum_{l \in \operatorname{IN~} f, h(t)} q_{l}(t) c_{e, l, h}(t)}{V_{f, h}(t)}, \\
& \mathrm{d}_{t} x_{h}(t)=\beta_{h} x_{h}(t),
\end{aligned}
$$

where $c_{e, l, h}(t)$ is the chlorine concentration in flow $l$ to tank $h$ at instant $t$.

It is obvious that the water transport through the tank does not occur for the case of a storage tank with separated water filling and drawing cycles during the drawing phase. Clearly, the chlorine concentration during the drawing phase decreases with time in accordance with kinetics decay (cf. (23)). As opposed to the drawing phase, the chlorine concentration dynamics in the tank during the filling phase depends on the chlorine concentration at all inflows to the tank (cf. (22)). Moreover, $c_{e, l, h}(t)$ is at the same time the chlorine concentration at the end of the last segment of the $l$-th pipe supplying water into the tank. Hence (22) may be rewritten as

$$
\begin{array}{r}
\mathrm{d}_{t} x_{h}(t)=\left(-\frac{S_{3}}{V_{f, h}(t)}+\beta_{h}\right) x_{h}(t) \\
+\frac{\sum_{l \in \mathrm{IN} f, h(t)} q_{l}(t) x_{l}(t)}{V_{f, h}(t)},
\end{array}
$$

where $x_{l}(t)$ is the state variable representing the chlorine concentration at the end of the last segment of the $l$-th pipe supplying water into tank $h$ at instant $t$. 
Finally, due to 21, 23-24 and taking (12) and (13) into account, the chlorine concentration dynamics throughout the entire DWDS can be written as

$$
\mathrm{d}_{t} \mathbf{x}(t)=\mathbf{A}(t) \mathbf{x}(t)+\mathbf{b}(t)
$$

where $\mathbf{A}(t)$ is the time-varying state matrix whose elements are composed of the hydraulic quantities, lengths of pipe segments and reaction rate coefficients; $\mathbf{b}(t)$ denotes the vector of inputs whose elements are dependent on the hydraulic quantities, the lengths of pipes segments, quality quantities at reservoirs and injection of the chlorine at the quality controlled nodes.

The water quality model described by (12)-13 and (25) was implemented in MATLAB. The model performance was examined by comparing its results with those obtained by applying the well-known EPANET simulator which serves as the virtual benchmark plant. The EPANET accurately integrates the quality model (1)-(4), (12) and (13) and the hydraulic model equations that are omitted in this paper in order to determine the flow rates $q(t)$ and linear water flow velocities $v(t)$ (Brdys and Ulanicki, 1994). The network quantity-quality model equations can be considered a faithful representation of reality. The model of water quality described by (2)-(4), (12) - (13) and (20) is viewed as the approximate model of reality. Comparative results for an example DWDS can be found in the work of Łangowski and Brdys (2007).

\section{Estimation of the chlorine concentration in the DWDS}

The dynamics of the chlorine concentration in the DWDS is described by model (25) and some special properties can be distinguished:

P1: The magnitudes and directions of the water flow rates determine the water quality model structure.

P2: The hydraulic quantities as well as the quality quantities are non-negative and bounded. Therefore

$$
\forall t \geq 0:\left\{\begin{array}{l}
\mathbf{0} \leq \mathbf{q}(t) \leq \mathbf{q}^{\max } \\
\mathbf{0} \leq \mathbf{v}(t) \leq \mathbf{v}^{\max } \\
\mathbf{0} \leq \mathbf{p}(t) \leq \mathbf{p}^{\max } \\
\mathbf{0} \leq \mathbf{c}(t) \leq \mathbf{c}^{\max }
\end{array}\right.
$$

where $\leq$ is understood element-wise; $\mathbf{q}^{\max }, \mathbf{v}^{\max }, \mathbf{p}^{\max }$, $\mathbf{c}^{\text {max }}$ denote the maximal values of flow rates, linear water flow velocities, pressures and chlorine concentrations in the DWDS, respectively.

P3: The chlorine concentrations only at certain network nodes are simultaneously state variables (the state is directly transferred to the system output). In this case, each of the output measurement equations can be written as

$$
\mathbf{y}_{c_{\text {out }}}(t)=\mathbf{x}_{2}(t) \text {, }
$$

where $\mathbf{y}_{c_{\text {out }}}(t)$ is the vector of measurements; $\mathbf{x}_{2}(t)$ denotes the vector of measurable state variables.

P4: The dynamics of the chlorine concentration in the DWDS described by 25 is cooperative. This is because the off-diagonal elements of the Jacobian matrix of the model (25) are non-negative. For example networks in the work of Łangowski and Brdys (2007) or Brdys and Łangowski (2008) this was shown by employing a cooperative system definition (Smith, 1995). It is worth adding that this property has a crucial meaning for the further design of the interval observer.

Notice that in order to solve the water quality model (cf. Section 2.1) the values of hydraulic quantities have to be available. This information is delivered by the quantity monitoring system. The monitoring system gathers information from the hydraulic sensors which are used in the DWDS. Typically, only a limited number of hydraulic quantities are directly measured in the DWDS due to, e.g., the sensors and their maintenance costs. Therefore, in order to recover a lack of hydraulic information, the necessity of employing a hydraulics estimation based on available hydraulic hard measurements and, e.g., hydraulics mathematical models arises. The hydraulics estimator based on set-membership uncertainty modelling can be found in the works of Brdys and Chen (1995), Duzinkiewicz (2006). Hence the hydraulics estimator delivers estimates of hydraulic quantities in an interval form. Clearly, the upper and lower trajectories of hydraulic quantity estimates are constant over each $T_{H}$. It is assumed that hydraulics information in this form is known for the DWDS considered but it is not obtained from the hydraulics estimator. In order to simulate the estimator, the EPANET is used. Assume that $T_{H}=1[\mathrm{~h}]$, $T=24[\mathrm{~h}]$ and for a nominal demand pattern hydraulic simulation results are obtained from the EPANET. Next, the values of hydraulic quantities are disturbed by about $\pm \Gamma \%$. Hence, the interval form of the hydraulic quantities is

$$
\begin{aligned}
q_{l}^{ \pm}(j) & =q_{l}(j) \pm \Gamma q_{l}(j), \\
v_{l}^{ \pm}(j) & =v_{l}(j) \pm \Gamma v_{l}(j), \\
V_{f, h}^{ \pm}(j) & =V_{f, h}(j) \pm \Gamma V_{f, h}(j),
\end{aligned}
$$

where \pm is to distinguish between the upper and the lower hydraulic quantity bounds; $q_{l}(j)$ and $v_{l}(j)$ denote the flow rate and the linear water flow velocity gathered from the EPANET for the $l$-th pipe at the $j$-th $T_{H}$, respectively; $V_{f, h}(j)$ stands for the $h$-th tank volume gathered from the EPANET at the $j$-th $T_{H} ; j=1,2,3, \ldots, J$ and $J=T / T_{H}=24[\mathrm{~h}] / 1[\mathrm{~h}]=24$.

In accordance with (28)-(30) the upper and the lower trajectories for the hydraulic quantities are constant over each $T_{H}$. Nevertheless, over the entire time horizon $T$, these trajectories can be treated as continuous and 
time-varying. The upper and the lower trajectories of the hydraulic quantities obtained in this way might be more conservative than trajectories that could be delivered by the hydraulics estimator. However, for the water quality estimation purposes, the hydraulic information obtained as above is sufficient. Moreover, it is assumed that the chlorine concentrations at the water sources are constant, but their values are not exactly known. Clearly, only certain upper $c_{s r}^{+}$and lower $c_{s r}^{-}$bounds on these quantities are available, so that for each water source we have

$$
c_{s r}^{-} \leq c_{s r} \leq c_{s r}^{+} .
$$

Because the hydraulic quantities as well as the quality at reservoirs are not exactly known, but certain upper and lower bounds are available, we have

$$
\begin{gathered}
\mathbf{b}^{-}(t) \leq \mathbf{b}(t) \leq \mathbf{b}^{+}(t), \\
\mathbf{A}^{-}(t) \leq \mathbf{A}(t) \leq \mathbf{A}^{+}(t)
\end{gathered}
$$

Remark 2. The trajectories of hydraulic quantities are constant over each $T_{H}$. Therefore, for successive $T_{H}$ the inequality (33) can be rewritten as

$$
\mathbf{A}^{-} \leq \mathbf{A} \leq \mathbf{A}^{+}
$$

U1: It is assumed that, although the state initial conditions are unknown, some bounds are available, so that

$$
\mathbf{x}^{-}(0) \leq \mathbf{x}(0) \leq \mathbf{x}^{+}(0)
$$

U2: The chlorine concentration measurements at the nodes are contaminated by the measurement uncertainty. Hence 27) is rewritten as

$$
\mathbf{y}_{c_{\text {out }}}(t)=\mathbf{x}_{2}(t)+\varepsilon_{c_{\text {out }}}(t),
$$

where $\varepsilon_{c_{\text {out }}}(t)$ is the measurement error.

It is assumed that $\varepsilon_{c_{\text {out }}}(t)$ is bounded:

$$
\left|\varepsilon_{c_{\text {out }}}(t)\right| \leq \varepsilon_{c_{\text {out }}}^{\max } .
$$

Hence the measured state can be bounded as

$$
\mathbf{x}_{2}^{-}(t) \leq \mathbf{x}_{2}(t) \leq \mathbf{x}_{2}^{+}(t),
$$

where $\mathbf{x}_{2}^{-}(t)=\mathbf{y}_{c_{\text {out }}}(t)-\varepsilon_{c_{\text {out }}}^{\max }$ and $\mathbf{x}_{2}^{+}(t)=\mathbf{y}_{c_{\text {out }}}(t)+$ $\varepsilon_{c_{\text {out }}}^{\max }$ and due to $\mathrm{P} 2$ we have $\mathbf{x}_{2}^{-}(t), \mathbf{x}_{2}(t), \mathbf{x}_{2}^{+}(t) \geq$ $\mathbf{0}, \forall t \geq 0$.

Note that the uncertainty in the system dynamics, inputs, initial conditions and measurement errors is described by a deterministic model. In particular, the set-membership model of uncertainty is used. Hence, in order to estimate the chlorine concentration in the DWDS, set bounded estimation can be applied.

\section{Interval observer for water quality in the DWDS}

In this section the possibility of applying the interval observer proposed by Alcaraz-González et al. (2005) to water quality estimation will be investigated. As has been assumed, the hydraulic quantities are not exactly known. Thus, the state matrix $\mathbf{A}(t)$ is uncertain (cf. (33)). Hence, in order to use the above-mentioned structure of the interval observer during estimation of the chlorine concentrations in the DWDS, the uncertainty in the matrix $\mathbf{A}(t)$ should be eliminated. It is possible to decompose the matrix $\mathbf{A}(t)$ as (Łangowski and Brdys, 2006)

$$
\mathbf{A}(t)=\overline{\mathbf{A}}(t)+\Lambda \mathbf{A}(t),
$$

where $\overline{\mathbf{A}}(t)$ is an exactly known part of the state matrix $\mathbf{A}(t) ; \Lambda \mathbf{A}(t)$ is the resulting uncertainty in the matrix $\mathbf{A}(t)$.

In order to apply the interval observer proposed by Alcaraz-González et al. (2005) to the DWDS, 25) may be rewritten as

$$
\mathrm{d}_{t} \mathbf{x}(t)=\overline{\mathbf{A}}(t) \mathbf{x}(t)+\overline{\mathbf{b}}(t)
$$

where

$$
\overline{\mathbf{b}}(t)=\Lambda \mathbf{A}(t) \mathbf{x}(t)+\mathbf{b}(t) .
$$

In order to determine the bounds on $\overline{\mathbf{b}}(t)$ needed for the observer design, the bounds $\mathbf{b}^{-}(t), \mathbf{b}^{+}(t)$ have to be modified in order to incorporate the uncertainty in $\Lambda \mathbf{A}(t) \mathbf{x}(t)$. The latter can be bounded by using a-priori bounds on $\Lambda \mathbf{A}(t)$ and $\mathbf{x}(t)$. Assuming that the state measurements (cf. P3) are exactly known and taking the above remarks into account, the DWDS interval observer is (Łangowski and Brdys, 2006)

$$
\left(\mathbf{S}^{ \pm}\right):\left\{\begin{aligned}
& \mathrm{d}_{t} \mathbf{w}^{ \pm}(t)= \overline{\mathbf{A}}_{11}(t) \mathbf{w}^{ \pm}(t) \\
&+\mathbf{N}_{1} \overline{\mathbf{A}}_{12}(t) \mathbf{x}_{2}(t)+\mathbf{M} \overline{\mathbf{v}}^{ \pm}(t) \\
& \mathbf{w}^{ \pm}(0)= \mathbf{N x}^{ \pm}(0) \\
& \hat{\mathbf{x}}_{1}^{ \pm}(t)=\mathbf{N}_{1}^{-1} \mathbf{w}^{ \pm}(t)
\end{aligned}\right.
$$

where $\mathbf{x}(t)=\left[\begin{array}{ll}\mathbf{x}_{1}(t) & \mathbf{x}_{2}(t)\end{array}\right]^{T} \in \mathbb{R}^{n}$ is the state vector and $\mathbf{x}_{1}(t) \in \mathbb{R}^{s}, \mathbf{x}_{2}(t) \in \mathbb{R}^{m}$ are the vectors of unmeasured and measured state variables, respectively and $s=n-m ; \hat{\mathbf{x}}_{1}^{ \pm}(t)$ denote the upper and lower bounds on the estimated state variables; $\mathbf{w}(t)$ is an auxiliary variable, defined as $\mathbf{w}(t)=\mathbf{N x}(t) ; \mathbf{N}=\left[\begin{array}{ll}\mathbf{N}_{1} & \mathbf{0}\end{array}\right] ; \mathbf{N}_{1}=$ $\eta \mathbf{I} \in \mathbb{R}^{s \times s}$ denotes an invertible matrix proportional to the identity matrix and $\eta$ is an arbitrary, positive and constant parameter; $\mathbf{M}=\left[\begin{array}{lll}\mathbf{N}_{1} & \mathbf{0} & \mathbf{0}\end{array}\right] ; \overline{\mathbf{v}}^{ \pm}(t)=$

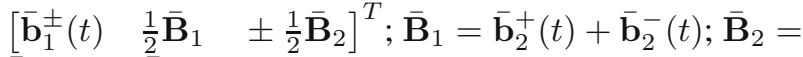
$\mathbf{b}_{2}^{+}(t)-\overline{\mathbf{b}}_{2}^{-}(t) ; \mathbf{A}_{11}(t)=\left\{a_{i, j}\right\}, \mathbf{A}_{12}(t)=\left\{a_{i, p}\right\}$, $\underline{\mathbf{A}_{21}(t)}=\left\{a_{p, j}\right\}, \mathbf{A}_{22}(t)=\left\{a_{p, p}\right\}, \forall i, j \in \overline{1, s}, \forall p \in$ $\overline{s+1, m}$ are suitable parts of the matrix $\mathbf{A}(t)$ structured 
by the measurement state variables

$$
\begin{aligned}
& \mathbf{A}(t) \\
& =\underset{\text { meas. }}{\text { est. }}\left[\begin{array}{cc}
\mathbf{A}_{11}(t) \in \mathbb{R}^{s \times s} & \mathbf{A}_{12}(t) \in \mathbb{R}^{s \times m} \\
\mathbf{A}_{21}(t) \in \mathbb{R}^{m \times s} & \mathbf{A}_{22}(t) \in \mathbb{R}^{m \times m}
\end{array}\right]_{n \times n}
\end{aligned}
$$

$\mathbf{b}_{1}(t)=\left\{b_{i}\right\}, \mathbf{b}_{2}(t)=\left\{b_{p}\right\}$ denote suitable parts of the vector $\mathbf{b}(t)$ structured by the measurement state variables:

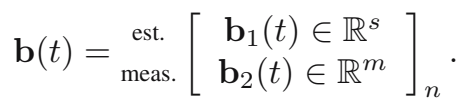

Observe that the matrix $\overline{\mathbf{A}}(t)$ and vector $\overline{\mathbf{b}}(t)$ are constructed analogously as $\mathbf{A}(t)$ and $\mathbf{b}(t)$.

The above interval observer may suffer from significant conservatism due to possibly excessive bounds on $\mathbf{x}(t)$. Also, the measurement uncertainty may not be negligible and, therefore, it is further developed. Starting from the DWDS dynamics described by (25), applying in the interval observer proposed by Alcaraz-González et al. (2005) bounds $\mathbf{A}^{+}(t), \mathbf{A}^{-}(t)$ instead of the known matrix $\overline{\mathbf{A}}(t)$ and bounds $\mathbf{x}_{2}^{+}(t), \mathbf{x}_{2}^{-}(t)$ (cf. U2) instead of $\mathbf{x}_{2}(t)$ that are based on the measurement error bounds, yields (Łangowski and Brdys, 2007; Brdys and Łangowski, 2008):

$$
\left(\mathbf{S}^{ \pm}\right):\left\{\begin{aligned}
& \mathrm{d}_{t} \mathbf{w}^{ \pm}(t)= \mathbf{A}_{11}^{ \pm}(t) \mathbf{w}^{ \pm}(t) \\
& \quad+\mathbf{N}_{1} \mathbf{A}_{12}^{ \pm}(t) \mathbf{x}_{2}^{ \pm}(t)+\mathbf{M} \mathbf{v}^{ \pm}(t) \\
& \mathbf{w}^{ \pm}(0)= \mathbf{N x}^{ \pm}(0) \\
& \hat{\mathbf{x}}_{1}^{ \pm}(t)=\mathbf{N}_{1}^{-1} \mathbf{w}^{ \pm}(t)
\end{aligned}\right.
$$

where $\mathbf{v}^{ \pm}(t)=\left[\begin{array}{lll}\mathbf{b}_{1}^{ \pm}(t) & \frac{1}{2} \mathbf{B}_{1} \quad \pm \frac{1}{2} \mathbf{B}_{2}\end{array}\right]^{T} ; \mathbf{B}_{1}=$ $\mathbf{b}_{2}^{+}(t)+\mathbf{b}_{2}^{-}(t) ; \mathbf{B}_{2}=\mathbf{b}_{2}^{+}(t)-\mathbf{b}_{2}^{-}(t) ; \mathbf{x}_{2}^{ \pm}(t)$ denote the upper and lower bounds on the measured state variables given by (36).

Remark 3. It is clear due to (32) and (33) that $\mathbf{A}_{11}^{-}(t) \leq$ $\mathbf{A}_{11}(t) \leq \mathbf{A}_{11}^{+}(t), \mathbf{A}_{12}^{-}(t) \leq \mathbf{A}_{12}(t) \leq \mathbf{A}_{12}^{+}(t), \mathbf{b}_{1}^{-}(t) \leq$ $\mathbf{b}_{1}(t) \leq \mathbf{b}_{1}^{+}(t)$ and $\mathbf{b}_{2}^{-}(t) \leq \mathbf{b}_{2}(t) \leq \mathbf{b}_{2}^{+}(t)$.

Theorem 1. (Brdys and Łangowski, 2008) The interval observer (41) produces stable and robust upper and lower envelopes $\hat{\mathbf{x}}_{1}^{+}(t)$ and $\hat{\mathbf{x}}_{1}^{-}(t)$, respectively, bounding the unmeasured state variables $\mathbf{x}_{1}(t)$ in spite of the uncertainty in inputs (chlorine measurements at reservoirs), initial conditions, state measurements (chlorine measurements at network nodes) and the state matrix $\mathbf{A}(t)$ in the linear part of the system dynamics.

Proof. The upper and the lower estimation errors can be written as

$$
\begin{gathered}
\mathbf{e}^{+}(t)=\hat{\mathbf{x}}_{1}^{+}(t)-\mathbf{x}_{1}(t), \\
\mathbf{e}^{-}(t)=\mathbf{x}_{1}(t)-\hat{\mathbf{x}}_{1}^{-}(t)
\end{gathered}
$$

for the systems $\mathbf{S}^{+}$and $\mathbf{S}^{-}$, respectively. Without any loss on generality, only $\mathbf{S}^{+}$will be further considered. Because of the directions of water flow the rates are constant only over each $T_{H}$, and the water quality model is also constant only over these intervals. Moreover, the magnitudes of hydraulic quantities are also constant over each $T_{H}$ (cf. $\mathrm{P} 1)$. Hence the state matrix $\mathbf{A}(t)$ is time-independent over each $T_{H}$. Therefore, the upper estimation error dynamics associated with $\mathbf{S}^{+}$over each $T_{H}$ is as follows:

$$
\begin{aligned}
\mathrm{d}_{t} \mathbf{e}^{+}(t)= & \underbrace{\mathbf{N}_{1}^{-1} \mathbf{A}_{11}^{+} \mathbf{N}_{1} \hat{\mathbf{x}}_{1}^{+}(t)-\mathbf{N}_{1}^{-1} \mathbf{A}_{11} \mathbf{N}_{1} \mathbf{x}_{1}(t)}_{\mathbf{R}^{+}(t)} \\
& +\mathbf{A}_{12}^{+} \mathbf{x}_{2}^{+}(t)-\mathbf{A}_{12} \mathbf{x}_{2}(t) \\
& +\mathbf{N}_{1}^{-1} \mathbf{M} \mathbf{v}^{+}(t)-\mathbf{N}_{1}^{-1} \mathbf{N b}(t) .
\end{aligned}
$$

First, the component $\mathbf{R}^{+}(t)$ will be considered. It is easy to show that

$$
\mathbf{R}^{+}(t)=\mathbf{A}_{11}^{+} \hat{\mathbf{x}}_{1}^{+}(t)-\mathbf{A}_{11} \mathbf{x}_{1}(t) .
$$

Owing to (34), we have

$$
\mathbf{A}_{11}^{+}-\mathbf{A}_{11}=\Delta \mathbf{A}_{11}^{+} \quad \Rightarrow \quad \mathbf{A}_{11}=\mathbf{A}_{11}^{+}-\Delta \mathbf{A}_{11}^{+} .
$$

Combining (46) with (45), we get

$$
\mathbf{R}^{+}(t)=\mathbf{A}_{11}^{+}\left(\hat{\mathbf{x}}_{1}^{+}(t)-\mathbf{x}_{1}(t)\right)+\Delta \mathbf{A}_{11}^{+} \mathbf{x}_{1}(t) .
$$

Combining 47 and (44), the dynamics of the upper estimation error become

$$
d_{t} \mathbf{e}^{+}(t)=\mathbf{A}_{11}^{+} \mathbf{e}^{+}(t)+\underbrace{\mathbf{I}+\mathbf{I I}-\mathbf{I I I}+\mathbf{I V}}_{\mathbf{H}^{+}(t)},
$$

where

$$
\begin{aligned}
\mathbf{I} & =\Delta \mathbf{A}_{11}^{+} \mathbf{x}_{1}(t), \\
\mathbf{I I} & =\mathbf{A}_{12}^{+} \mathbf{x}_{2}^{+}(t), \\
\mathbf{I I I} & =\mathbf{A}_{12} \mathbf{x}_{2}(t), \\
\mathbf{I V} & =\mathbf{N}_{1}^{-1} \mathbf{M} \mathbf{v}^{+}(t)-\mathbf{N}_{1}^{-1} \mathbf{N b}(t) .
\end{aligned}
$$

Owing to P2, P4 and Remark 3 we have

$$
\mathbf{A}_{11, i j}^{+} \geq 0, \quad \forall i \neq j .
$$

Hence the system (48) has cooperative dynamics. Moreover, the system $\mathbf{S}^{+}$also has cooperative dynamics (Smith, 1995). Additionally, using the definitions of the upper estimation error $\mathbf{e}^{+}(t)$, the structure of $\mathbf{S}^{+}$and $\mathbf{U} 1$, it is easy to show that

$$
\mathbf{e}^{+}(0)=\hat{\mathbf{x}}_{1}^{+}(0)-\mathbf{x}_{1}(0) \geq \mathbf{0}
$$

We shall now check the sign of $\mathbf{H}^{+}(t)$. Due to (46), Remark 3 and P2 it is clear that $\mathbf{I}=\Delta \mathbf{A}_{11}^{+} \mathbf{x}_{1}(t) \geq \mathbf{0}$. Moreover, using Remark 3, P2 and P4: II $=\mathbf{A}_{12}^{+} \mathbf{x}_{2}^{+}(t) \geq$ 
$\mathbf{0}$ and $\mathbf{I I I}=\mathbf{A}_{12}(t) \mathbf{x}_{2}(t) \geq \mathbf{0}$. By Remark 3 and U2 the inequality $\mathbf{A}_{12}^{+} \mathbf{x}_{2}^{+}(t)-\mathbf{A}_{12}(t) \mathbf{x}_{2}(t) \geq \mathbf{0}$ is ensured. The non-negative sign of IV is ensured by P2, Remark 3 and the choice of $\mathbf{N}_{1}$. As I, II, III and IV are non-negative, so is $\mathbf{H}^{+}(t)$. Moreover, as P2 holds, $\mathbf{H}^{+}(t)$ is bounded. Due to the properties of linear cooperative systems (Hadj-Sadok and Gouzé, 2001), the upper estimation error satisfies

$$
\mathbf{e}^{+}(t) \geq \mathbf{0}, \quad \forall t>0 .
$$

Hence the trajectories of the system $\mathbf{S}^{+}$dominate the trajectories of (25) from above. Clearly, $\mathbf{S}^{+}$generates the upper bounds on the estimated state variables.

Let us now examine the stability of the interval observer (41). If $\mathbf{A}_{11}^{+}$is Hurwitz stable then the dynamics of the upper estimation error (42) is also stable. It was checked for the DWDS presented in Section 5 as well as for example DWDSs considered by Łangowski and Brdys (2007) or Brdys and Łangowski (2008) that $\mathbf{A}_{11}^{+}$is truly Hurwitz stable over each $T_{H}$. Moreover, they proved that the matrix $\mathbf{A}_{11}^{-}$is also Hurwitz stable over each $T_{H}$.

Hence, the interval observer (41) is a robust and stable estimator for water quality estimation in the DWDS and it is guaranteed that $\hat{\mathbf{x}}_{1}^{-}(t) \leq \mathbf{x}_{1}(t) \leq \hat{\mathbf{x}}_{1}^{+}(t)$ for the initial conditions satisfying $\mathbf{x}^{-}(0) \leq \mathbf{x}(0) \leq \mathbf{x}^{+}(0)$.

The interval observer (41) requires state measurements. Unfortunately, the chlorine concentration measurements are the state measurements only at certain network nodes (cf. P3). Hence the proposed interval observer is further developed in order to handle a case where the chlorine concentration sensor is located at the node with several connected pipes. Additionally, it is assumed that the measuring node is the quality controlled one. Just for the sake of simplicity, with no loss of generality, a quality controlled node $n$ with two supplying pipes is considered. According to 12 the chlorine concentration at this node is

$$
y_{c_{\text {out }, n}}(t)=c_{\mathrm{in}, n}(t)+\frac{y_{q_{1}}(t) x_{1}(t)+y_{q_{2}}(t) x_{2}(t)}{y_{q_{1}}(t)+y_{q_{2}}(t)},
$$

where $y_{q_{1}}(t), y_{q_{2}}(t)$ are the measurements of water flow rates in pipes 1 and 2, respectively.

In this case the output measurement (27) is

$$
\mathbf{y}_{c_{\text {out }}}(t)=\overline{\mathbf{x}}_{2}(t)
$$

where

$$
\overline{\mathbf{x}}_{2}(t)=\left[\begin{array}{ll}
\mathbf{x}_{2}(t) & \widetilde{\mathbf{x}}_{2}(t)
\end{array}\right]^{T},
$$

and $\widetilde{\mathbf{x}}_{2}(t)$ denotes the vector of indirectly measured state variables and is called the vector of pseudo-measurements.

The measurements of $y_{c_{\mathrm{out}, n}}(t)$ cannot be directly utilised by the observer (41) as they are not measurements of the state variable. However, from (51) the state variable $x_{2}(t)$ can be expressed as

$$
\begin{aligned}
x_{2}(t)= & \frac{y_{q_{1}}(t)}{y_{q_{2}}(t)}\left(y_{c_{\mathrm{out}, n}}(t)-c_{\mathrm{in}, n}(t)\right) \\
& +y_{c_{\mathrm{out}, n}}(t)-c_{\mathrm{in}, n}(t)-\frac{y_{q_{1}}(t)}{y_{q_{2}}(t)} x_{1}(t),
\end{aligned}
$$

and used to define pseudo-measurements as

$$
\begin{aligned}
\widetilde{x}_{2}(t)= & \frac{y_{q_{1}}(t)}{y_{q_{2}}(t)}\left(y_{c_{\mathrm{out}, n}}(t)-c_{\mathrm{in}, n}(t)\right) \\
& +y_{c_{\mathrm{out}, n}}(t)-c_{i n, n}(t)-\frac{y_{q_{1}}(t)}{y_{q_{2}}(t)} \hat{x}_{1}(t) .
\end{aligned}
$$

Hence, in order to define the pseudo-measurements, the estimates of state variable $\hat{x}_{1}(t)$ are used instead of the state variable $x_{1}(t)$. Assuming that the chlorine concentration $c_{\mathrm{in}, n}(t)$ added to node $n$ is exactly known, the needed upper and the lower bounds on $\widetilde{x}_{2}(t)$ are calculated from (55):

$$
\begin{aligned}
\widetilde{x}_{2}^{+}(t)= & \frac{y_{q_{1}}^{+}(t)}{y_{q_{2}}^{-}(t)}\left(y_{c_{\mathrm{out}, n}}^{+}(t)-c_{\mathrm{in}, n}(t)\right) \\
& +y_{c_{\mathrm{out}, n}}^{+}(t)-c_{\mathrm{in}, n}(t)-\frac{y_{q_{1}}^{-}(t)}{y_{q_{2}}^{+}(t)} \hat{x}_{1}^{-}(t) \\
\widetilde{x}_{2}^{-}(t)= & \frac{y_{q_{1}}^{-}(t)}{y_{q_{2}}^{+}(t)}\left(y_{c_{\mathrm{out}, n}}^{-}(t)-c_{\mathrm{in}, n}(t)\right) \\
& +y_{c_{\mathrm{out}, n}}^{-}(t)-c_{\mathrm{in}, n}(t)-\frac{y_{q_{1}}^{+}(t)}{y_{q_{2}}^{-}(t)} \hat{x}_{1}^{+}(t) .
\end{aligned}
$$

If the vector of chlorine concentration measurements are defined as (52), then taking (56) into account, the following new structure of the interval observer is proposed:

$$
\left(\mathbf{S}^{ \pm}\right):\left\{\begin{aligned}
& \mathrm{d}_{t} \mathbf{w}^{ \pm}(t)= \mathbf{A}_{11}^{ \pm}(t) \mathbf{w}^{ \pm}(t) \\
&+\mathbf{N}_{1} \mathbf{A}_{12}^{ \pm}(t) \overline{\mathbf{x}}_{2}^{ \pm}(t)+\mathbf{M} \mathbf{v}^{ \pm}(t), \\
& \mathbf{w}^{ \pm}(0)= \mathbf{N x}^{ \pm}(0) \\
& \hat{\mathbf{x}}_{1}^{ \pm}(t)=\mathbf{N}_{1}^{-1} \mathbf{w}^{ \pm}(t) .
\end{aligned}\right.
$$

Note that if an uncontrolled node with more than one supplying pipe is considered, the term $c_{\mathrm{in}, n}(t)$ in the right-hand side of (56) disappears.

In order to estimate the unmeasured state variables by using the interval observer (57), the necessity of calculating the pseudo-measurements appears. Hence, the following procedure is proposed:

S1: The estimation of unmeasured state variables is performed by using the interval observer (41). During this estimation process only direct state measurements are used. 
S2: The pseudo-measurements of indirectly measured state variables are calculated by employing (56). Next, the state variables that refer to pseudo-measurements are removed from the estimated states and the vector of measurements $\mathbf{x}_{2}(t)$ is augmented by adding the vector of pseudo-measurements $\widetilde{\mathbf{x}}_{2}(t)$ to produce the interval observer (57).

Notice that the pseudo-measurements are the new, important source of information. Hence, the two-step estimation algorithm has a better performance than the estimation process without the indirect state measurements.

Theorem 2. The interval observer (57) produces stable and robust the upper and the lower envelopes $\hat{\mathbf{x}}_{1}^{+}(t)$ and $\hat{\mathbf{x}}_{1}^{-}(t)$, respectively, bounding the unmeasured state variables $\mathbf{x}_{1}(t)$ in spite of the uncertainty in inputs, initial conditions, direct and indirect state measurements and the state matrix $\mathbf{A}(t)$ in the linear part of the system dynamics.

Proof. It is obvious that the upper and the lower estimation errors are still described by (42) and (43). Therefore, the upper estimation error dynamics is still defined by (48), where $\mathbf{I}=\Delta \mathbf{A}_{11}^{+} \mathbf{x}_{1}(t), \mathbf{I I}=\mathbf{A}_{12}^{+} \overline{\mathbf{x}}_{2}^{+}(t)$, III $=$ $\mathbf{A}_{12}(t) \overline{\mathbf{x}}_{2}(t), \mathbf{I V}=\mathbf{N}_{1}^{-1} \mathbf{M} \mathbf{v}^{+}(t)-\mathbf{N}_{1}^{-1} \mathbf{N} \mathbf{b}(t)$. Since the upper estimation error dynamics are still determined by the matrix $\mathbf{A}_{11}^{+}$, the system (48) has cooperative dynamics. Hence the dynamics of the interval observer (57) remains also cooperative.

We shall now check the sign of $\mathbf{H}^{+}(t)$. Observe that the structure of $\mathbf{H}^{+}(t)$ has one difference. Clearly, the vector of measurements is composed of the state measurements and pseudo-measurements. Hence, the inequality $\mathbf{x}_{2}^{-}(t) \leq \mathbf{x}_{2}(t) \leq \mathbf{x}_{2}^{+}(t)$ is ensured by U2 and due to $\mathrm{P} 2$ it is obvious that $\mathbf{x}_{2}^{-}(t), \mathbf{x}_{2}(t), \mathbf{x}_{2}^{+}(t)$ $\geq \mathbf{0}, \forall t \geq 0$. However, it is necessary to show that the following holds:

$$
\begin{aligned}
& \widetilde{\mathbf{x}}_{2}^{-}(t) \leq \widetilde{\mathbf{x}}_{2}(t) \leq \widetilde{\mathbf{x}}_{2}^{+}(t), \\
& \widetilde{\mathbf{x}}_{2}^{-}(t), \widetilde{\mathbf{x}}_{2}(t), \widetilde{\mathbf{x}}_{2}^{+}(t) \geq \mathbf{0}, \quad \forall t \geq 0 .
\end{aligned}
$$

We shall now prove that the inequality $(58)$ is really fulfilled. First, since $\hat{x}_{1}(t) \in\left[\hat{x}_{1}^{-}(t), \hat{x}_{1}^{+}(t)\right]$ is in fact unknown, the Chebyshev centre $\hat{x}_{1}^{*}(t)$ of the enveloped estimates is to be used. Hence, (55) can be rewritten as

$$
\begin{aligned}
\widetilde{x}_{2}(t)= & \frac{y_{q_{1}}(t)}{y_{q_{2}}(t)}\left(y_{c_{\mathrm{ou}, n}}(t)-c_{\mathrm{in}, n}(t)\right) \\
& +y_{c_{\mathrm{out}, n}}(t)-c_{\mathrm{in}, n}(t)-\frac{y_{q_{1}}(t)}{y_{q_{2}}(t)} \hat{x}_{1}^{*}(t)
\end{aligned}
$$

Due to P2, (33), U2 and since the interval observer (41) is robust and stable, we get

$$
\begin{aligned}
y_{q_{1}}^{-}(t) & \leq y_{q_{1}}(t) \leq y_{q_{1}}^{+}(t), \\
y_{q_{2}}^{-}(t) & \leq y_{q_{2}}(t) \leq y_{q_{2}}^{+}(t), \\
y_{c_{\mathrm{out}, n}^{-}}^{-}(t) & \leq y_{c_{\mathrm{out}, n}}(t) \leq y_{c_{\mathrm{out}, n}}^{+}(t), \\
\hat{x}_{1}^{-}(t) & \leq \hat{x}_{1}^{*}(t) \leq \hat{x}_{1}^{+}(t),
\end{aligned}
$$

$$
\begin{aligned}
& y_{q_{1}}^{-}(t), y_{q_{1}}(t), y_{q_{1}}^{+}(t), y_{q_{2}}^{-}(t), y_{q_{2}}(t), y_{q_{2}}^{+}(t), \\
& y_{c_{\mathrm{out}, n}}^{-}(t), y_{c_{\mathrm{out}, n}}(t), y_{c_{\mathrm{out}, n}}^{+}(t), \\
& \hat{x}_{1}^{-}(t), \hat{x}_{1}^{*}(t), \hat{x}_{1}^{+}(t), c_{\mathrm{in}, n}(t) \geq 0, \quad \forall t \geq 0 .
\end{aligned}
$$

Hence, due to 60 and taking into account the mutual relations between $y_{\mathcal{c}_{\mathrm{out}, n}}^{-}(t), y_{c_{\mathrm{out}, n}}(t), y_{c_{\mathrm{out}, n}}^{+}(t)$ and $\hat{x}_{1}^{-}(t), \hat{x}_{1}^{*}(t), \hat{x}_{1}^{+}(t)$, this can be written as

$$
\begin{aligned}
\widetilde{x}_{2}^{+}(t)= & \frac{y_{q_{1}}^{+}(t)}{y_{q_{2}}^{-}(t)}\left(y_{c_{\mathrm{out}, n}}^{+}(t)-c_{\mathrm{in}, n}(t)\right) \\
& +y_{c_{\mathrm{out}, n}^{+}}^{+}(t)-c_{\mathrm{in}, n}(t)-\frac{y_{q_{1}}^{-}(t)}{y_{q_{2}}^{+}(t)} \hat{x}_{1}^{-}(t) \geq 0 .
\end{aligned}
$$

Unfortunately, it is possible that $\widetilde{x}_{2}(t)$ and $\widetilde{x}_{2}^{-}(t)$ will be negative. However, because $\mathrm{P} 2$ holds, these cases are eliminated. Clearly, it is assumed that if $\widetilde{x}_{2}(t)$ and $\widetilde{x}_{2}^{-}(t)$ are negative, then during the estimation process the zero values for $\widetilde{x}_{2}(t)$ and $\widetilde{x}_{2}^{-}(t)$ are used. Therefore

$$
\begin{aligned}
\widetilde{x}_{2}(t)= & \frac{y_{q_{1}}(t)}{y_{q_{2}}(t)}\left(y_{c_{\mathrm{ou}, n}}(t)-c_{\mathrm{in}, n}(t)\right) \\
& +y_{c_{\mathrm{out}, n}}(t)-c_{\mathrm{in}, n}(t)-\frac{y_{q_{1}}(t)}{y_{q_{2}}(t)} \hat{x}_{1}^{*}(t) \geq 0, \\
\widetilde{x}_{2}^{-}(t)= & \frac{y_{q_{1}}^{-}(t)}{y_{q_{2}}^{+}(t)}\left(y_{c_{\mathrm{out}, n}^{-}}(t)-c_{i n, n}(t)\right) \\
& +y_{c_{\mathrm{out}, n}^{-}}(t)-c_{\mathrm{in}, n}(t)-\frac{y_{q_{1}}^{+}(t)}{y_{q_{2}}^{-}(t)} \hat{x}_{1}^{+}(t) \geq 0 .
\end{aligned}
$$

From 61 -63 it follows that $\widetilde{\mathbf{x}}_{2}^{-}(t), \widetilde{\mathbf{x}}_{2}(t), \widetilde{\mathbf{x}}_{2}^{+}(t) \geq \mathbf{0}$ $\forall t \geq 0$. Moreover, due to the above it is easy to show that $\widetilde{\mathbf{x}}_{2}^{-}(t) \leq \widetilde{\mathbf{x}}_{2}(t) \leq \widetilde{\mathbf{x}}_{2}^{+}(t)$. Furthermore, (58) implies that the initial conditions $\mathbf{e}^{+}(0)$ are non-negative and the sign of $\mathbf{H}^{+}(t)$ is also non-negative. Hence, the upper estimation error associated with the interval observer (57) satisfies (50). Therefore, the trajectories of the system (57) dominate the trajectories of the system (25) from above. Moreover, the interval observer (57) remains stable. Hence, the interval observer (57) is a robust and stable estimator for water quality estimation in the DWDS and it is guaranteed that $\hat{\mathbf{x}}_{1}^{-}(t) \leq \mathbf{x}_{1}(t) \leq \hat{\mathbf{x}}_{1}^{+}(t)$ for the initial conditions satisfying $\mathbf{x}^{-}(0) \leq \mathbf{x}(0) \leq \mathbf{x}^{+}(0)$.

Remark 4. Notice that the interval observers 411 and (57) are stable and robust over each hydraulic step $T_{H}$. 
Because the water flow directions and their magnitudes can change over a given time horizon, the water quality model structure can change as well (cf. Section 2). As a consequence, the interval observers have to be modified. In other words, for each $T_{H}$ a different structure of the interval observer, determined by the hydraulic quantities and their values, needs to be used. Therefore, the overall observer can be viewed as a mechanism with switched dynamics. Due to the stability of the observer dynamics, the switching time instants should be chosen carefully. In this paper the switches take place together with the successive $T_{H}$, which are inherited from the known hydraulic solution. Such an approach gives a structural consistency of the observer dynamics and the dynamics of the estimated process. Hence the switching is basically driven by the changes in the hydraulics and they can have impact on every nodes in the DWDS. Moreover, despite the knowledge of when the switching will follow, the structure of the next observer will be known from the time instant when a hydraulic solution for the next $T_{H}$ will be available. Therefore, the switching sequence can be hard to determine, as it depends on the operating states and conditions of the DWDS. Indeed, in order to ensure the observer stability over the entire time horizon, certain conditions constraining the switching time instants have to be added. In this paper the stability of the switching process has been investigated only by simulation.

The structures of the interval observers (41) and (57) require determination of the matrices $\mathbf{A}^{-}$and $\mathbf{A}^{+}$ over each $T_{H}$. Notice that the majority of elements in these matrices are directly composed of the pipe segment lengths, reaction rate coefficients and hydraulic quantities. However, certain matrices elements are evaluated by solving suitable optimisation problems. This situation occurs for the state variables representing the chlorine concentrations at the ends of pipe segments where the segments quality input equals the chlorine concentration at the DWDS node (cf. (iv)). This issue and formulation of suitable optimisation problems were described in detail by Łangowski and Brdys (2007). Moreover, by the definition of the state variables, the observers (41) and (57) return the quality estimates only at the DWDS nodes that are supplied by one pipe. By contrast, the chlorine concentrations at the nodes with several connected pipes are linear combinations of the appropriate state variables. Therefore, the chlorine concentrations at these nodes are calculated based on the estimates of these state variables by solving suitable optimisation problems. The formulation of these optimisation problems was described in detail by Łangowski and Brdys (2007).

Remark 5. It is obvious that for on-line estimation the efficiency of the estimation algorithm should be high. Notice that the interval estimation algorithm does not require solving on-line highly nonlinear and nonconvex optimisation problems during the estimation process. The estimates of unmeasured state variables result from solving systems of ordinary differential equations. Hence the efficiency of the interval estimation algorithm is high. This was examined for example DWDSs and corroborating results were discussed by Łangowski and Brdys (2007).

The proposed methodology for the model of free chlorine and DBPs concentrations has been further developed by Arminski and Brdys (2013).

\section{Case study}

The interval observers (41) and 57) were applied to the model of a real DWDS. The DWDS considered (cf. Fig. 1) is located in Chojnice in Poland. This DWDS delivers water to about 40,000 inhabitants (Nowicki et al., 2012). The numbers of particular elements in the DWDS are 177 nodes, 271 pipes, 2 reservoirs, 1 tank and 3 pumps. The water is provided to the network from two sources, which are modelled as reservoirs of treated water. There are not water quality controlled nodes. During modelling the DWDS was divided into seven parts and one cumulated consumer was assigned to each part. Consequently, there are seven water demand nodes. These water demand nodes are clearly marked in Fig. 1. The demand values as well as the demand patterns at these nodes are based on real data and two types of the demand pattern are distinguished: 1 and 2 (cf. Fig. 2). The demand pattern 1 is used at nodes $31,39,67,83$ and 88 , whereas the demand pattern 2 is used at nodes 60 and 70 .

The demand patterns (cf. Fig. 2) are used in order to obtain the nominal values of hydraulic quantities from the EPANET and then they are distorted by about $\pm 2 \%$. The chlorine concentration measurements and the chlorine concentration at reservoirs are provided also by the EPANET; however, reaction rate coefficients $\alpha$ and $\beta$ were changed. The chlorine concentration measurements are contaminated by the measurement error (cf. (27) of $\pm 2 \%$. According to Section $2.1 T_{Q \text {,min }}$ as well as $N_{l, \max }$ are introduced into the algorithm and they equal 4 [min] and 8, respectively. The estimation results at several nodes are shown in Figs. 3-6. Four trajectories are presented in each figure: the chlorine concentration from the EPANET cf., bounds on the estimated chlorine concentrations and Chebyshev centres.

The presented simulation results show that the chlorine concentration trajectories are always inside the estimated bounds. However, the abrupt changes in the trajectories during transients can be observed. This is the effect of initial and boundary conditions on the chlorine concentration being equal to zero at the beginning of the 


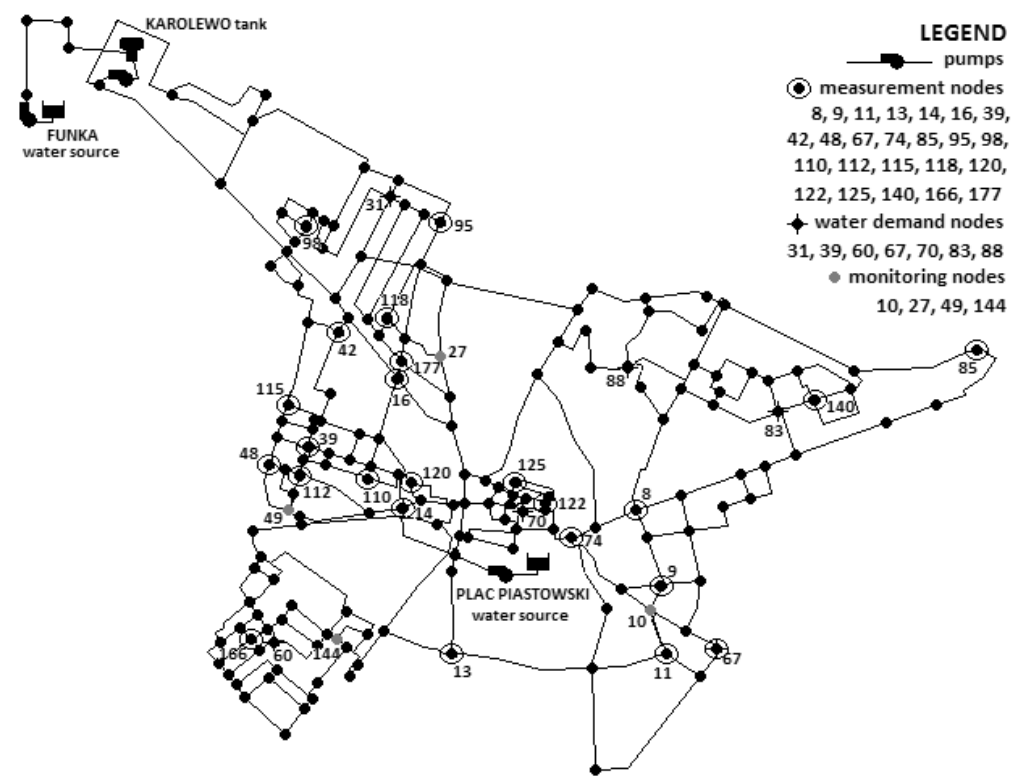

Fig. 1. Chojnice drinking water distribution system.

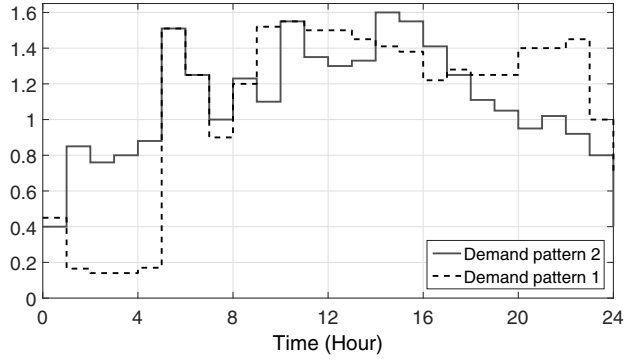

Fig. 2. Demand patterns at nodes 31, 39, 67, 83, 88, 60 and 70.

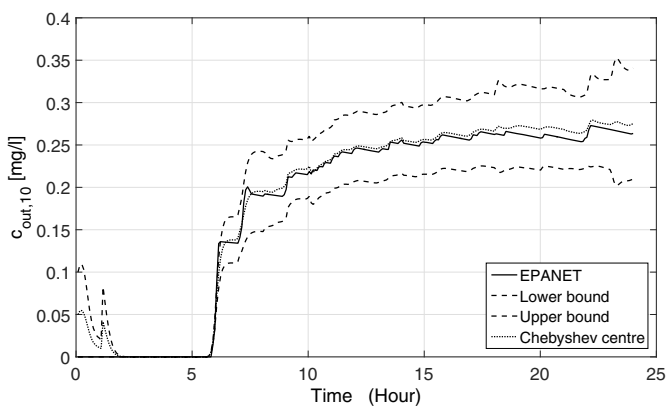

Fig. 3. Interval estimation results at node 10.

observation. The overall robust estimation results yield a satisfying performance.

\section{Conclusions}

In this paper a water quality model for estimation has been derived and implemented in MATLAB. Set bounded

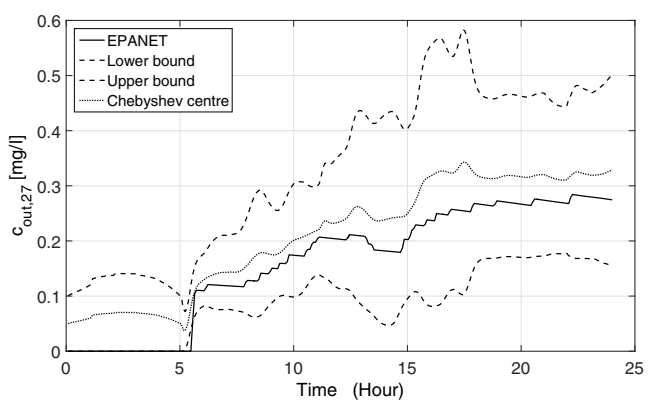

Fig. 4. Interval estimation results at node 27.

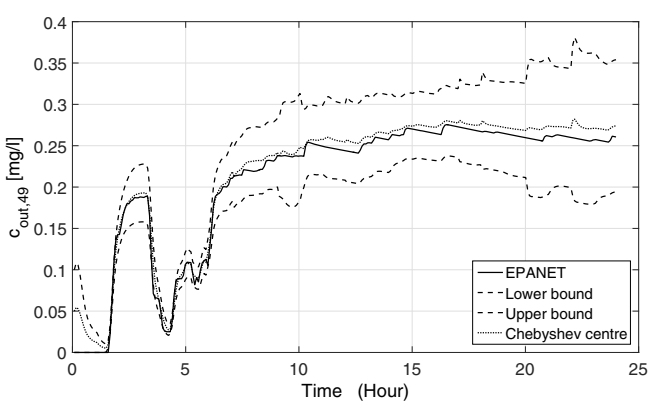

Fig. 5. Interval estimation results at node 49 .

estimation using an interval observer for the chlorine monitoring in the DWDS has been presented. The stability and robustness of the designed interval observer have been rigorously proved.

The proposed interval observer has been applied to the model of a real DWDS. The obtained results yield 


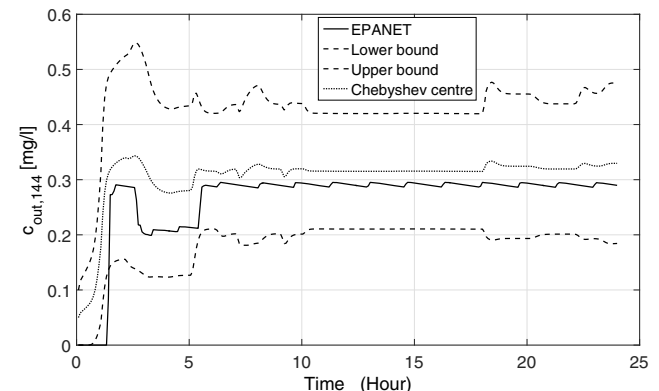

Fig. 6. Interval estimation results at node 144.

satisfying performance of the estimated bounds.

Some directions for future research are, i.a., deriving sufficient conditions for the stability and robustness of the interval observer during the switching time instants and developing additional mechanisms giving tighter estimation bounds.

\section{References}

Al-Omari, A.S. and Chaudhry, M.H. (2001). Unsteady-state inverse chlorine modeling in pipe networks, Journal of Hydraulic Engineering 127(8): 669-677.

Alcaraz-González, V., Harmand, J., Rapaport, A., Steyer, J.P., González-Alvarez, V. and Ortiz, C.P. (2005). Application of a robust interval observer to an anaerobic digestion process, Developments in Chemical Engineering and Mineral Processing 13(3-4): 267-278.

Amairi, M. (2016). Recursive set membership estimation for output-error fractional models with unknown-but-bounded errors, International Journal of Applied Mathematics and Computer Science 26(3): 543-553, DOI: 10.1515/amcs-2016-0038.

Arminski, K. and Brdys, M.A. (2013). Robust monitoring of water quality in drinking water distribution system, Proceedings of the 13th IFAC Symposium on Large Scale Complex Systems: Theory and Applications, Shanghai, China, Vol. 46, pp. 105-110.

Arminski, K., Zubowicz, T. and Brdys, M.A. (2013). A biochemical multi-species quality model of a drinking water distribution system for simulation and design, International Journal of Applied Mathematics and Computer Science 23(3): 571-585, DOI: 10.2478/amcs-2013-0043.

Boccelli, D.L., Tryby, M.E., Uber, J.G. and Summers, R.S. (2003). A reactive species model for chlorine decay and THM formation under rechlorination conditions, Water Research 37(11): 2654-2666.

Boulos, P.F., Lansey, K.E. and Karney, B.W. (2004). Comprehensive Water Distribution Systems Analysis Handbook, MWH Soft, Inc., Pasadena, CA.

Brdys, M.A. and Chen, K. (1995). Joint estimation of state and parameters in quantity models of water supply and distribution systems, Automatisierungstechnik 43(2): $77-84$
Brdys, M.A. and Chen, K. (1996). Joint estimation of states and parameters of integrated quantity and quality models of dynamic water supply and distribution systems, Proceedings of the 13th IFAC World Congress, San Francisco, CA, Vol. 1, pp. 759-762.

Brdys, M.A. and Łangowski, R. (2008). Interval estimator for chlorine monitoring in drinking water distribution systems under uncertain system dynamics, inputs and state measurement errors, Proceedings of the 11th IFAC/IFORS/IMACS/IFIP Symposium on Large Scale Systems: Theory and Applications, Gdańsk, Poland, Vol. 11, pp. 85-90.

Brdys, M.A., Puta, H., Arnold, E., Chen, K. and Hopfgarten, S. (1995). Operational control of integrated quality and quantity in water systems, Proceedings of the IFAC/IFORS/IMACS Symposium on Large Scale Systems, London, UK, Vol. 2, pp. 715-719.

Brdys, M.A. and Ulanicki, B. (1994). Operational Control of Water Systems: Structures, Algorithms and Applications, New York, NY.

Chen, K. (1997). Set Membership Estimation of State and Parameters and Operational Control of Integrated Quantity and Quality Models of Water Supply and Distribution Systems, $\mathrm{PhD}$ thesis, University of Birmingham, Birmingham.

Clark, R.M., Abdesaken, F., Boulos, P.F. and Mau, R.E. (1996). Mixing in distribution system storage tanks: Its effect on water quality, Journal of Environmental Engineering 122(9): 814-821.

Duzinkiewicz, K. (2006). Set membership estimation of parameters and variables in dynamic networks by recursive algorithms with a moving measurement window, International Journal of Applied Mathematics and Computer Science 16(2): 209-217.

Efimov, D. and Raïssi, T. (2016). Design of interval observers for uncertain dynamical systems, Automation and Remote Control 77(2): 191-225.

EU Cost Action IC0806-IntelliCIS (2008). Memorandum of Understanding, 7th Framework Program, http://www.intellicis.eu

Gouzé, J.L., Rapaport, A. and Hadj-Sadok, M.Z. (2000). Interval observers for uncertain biological systems, Ecological Modelling 133(1-2): 45-56.

Hadj-Sadok, M. Z. and Gouzé, J. L. (2001). Estimating of uncertain models of activated sludge process with interval observers, Journal of Process Control 11(3): 299-310.

Jauberthie, C., Travé-Massuyès, L. and Verdière, N. (2016). Set-membership identifiability of nonlinear models and related parameter estimation properties, International Journal of Applied Mathematics and Computer Science 26(4): 803-813, DOI: 10.1515/amcs-2016-0057.

Łangowski, R. (2015). Algorithms of Allocating Quality Sensors for Monitoring Quality in Drinking Water Distributions Systems, PhD thesis, Gdańsk University of Technology, Gdańsk, (in Polish). 
Łangowski, R. and Brdys, M.A. (2006). Interval asymptotic estimator for chlorine monitoring in drinking water distribution systems, Proceedings of the 1st IFAC Workshop on Applications of Large Scale Industrial Systems, Helsinki, Finland, Vol. 39, pp. 35-40.

Łangowski, R. and Brdys, M.A. (2007). Monitoring of chlorine concentration in drinking water distribution systems using interval estimator, International Journal of Applied Mathematics and Computer Science 17(2): 199-216, DOI: 10.2478/v10006-007-0019-y.

Males, R.M., Grayman, W.M. and Clark, R.M. (1988). Modeling water quality in distribution systems, Journal of Water Resources Planning and Management 114(2): 197-209.

Mau, R.E., Boulos, P.F., Clark, R.M., Grayman, W.M., Tekippe, R.J. and Trussell, R.R. (1995). Explicit mathematical models of distribution storage water quality, Journal of Hydraulic Engineering 121(10): 699-709.

Mitchel, A.R. and Griffiths, D.F. (1980). The Finite Difference Method in Partial Differential Equations, Wiley, Chichester.

Nowicki, A., Grochowski, M. and Duzinkiewicz, K. (2012). Data-driven models for fault detection using kernel PCA: A water distribution system case study, International Journal of Applied Mathematics and Computer Science 22(4): 939-949, DOI: 10.2478/v10006-012-0070-1.

Park, K. and Kuo, A.Y. (1996). A multi-step computation scheme: Decoupling kinetic processes from physical transport in water quality models, Water Research 30(10): 2255-2264.

Propato, M., Uber, J.G., Shang, F. and Polycarpou, M.M. (2001). Integrated control and booster system design for residual maintenance in water distribution systems, Proceedings of the World Water and Environmental Resources Congress, Orlando, FL, USA, Vol. 111, pp. 1-10.

Rapaport, A. and Dochain, D. (2005). Interval observers for biochemical processes with uncertain kinetics and inputs, Mathematical Biosciences 193(2): 235-253.

Rossman, L.A. and Boulos, P.F. (1996). Numerical methods for modeling water quality in distribution systems: A comparison, Journal of Water Resources Planning and Management 122(2): 137-146.

Rossman, L.A., Boulos, P.F. and Altman, T. (1993). Discrete volume-element method for network water-quality models, Journal of Water Resources Planning and Management 119(5): 505-517.

Rossman, L.A., Clark, R.M. and Grayman, W.M. (1994). Modeling chlorine residuals in drinking water distribution systems, Journal of Environmental Engineering 120(4): 803-820.

Smith, H.L. (1995). Monotone Dynamical Systems. An Introduction to the Theory of Competitive and Cooperative Systems, AMS, Providence, RI.

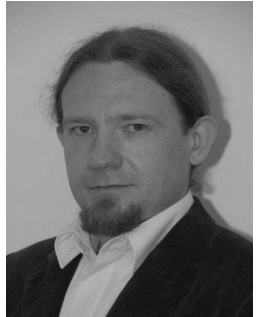

Rafał Langowski received the $\mathrm{MSc}$ and the $\mathrm{PhD}$ degrees in control engineering (with honours) from the Faculty of Electrical and Control Engineering at the Gdańsk University of Technology in 2003 and 2015, respectively. From 2007 to 2014 he held the positions of a specialist and a manager at ENERGA, one of the biggest energy enterprises in Poland. Since February 2014 he has been an owner of the VIDEN company, working in the energy and control areas. He offers theoretical and practical experience, especially in front and back offices at energy companies and operation of the energy market in Poland. $\mathrm{He}$ is proficient in regulations as well as the processing of switching processes based on third party access and design of back office algorithms. From 2016 to 2017 he was a senior lecturer in the Department of Control Systems Engineering at the Gdańsk University of Technology. Currently he is an assistant professor there. His research interests involve mathematical modelling, estimation and monitoring of large scale complex systems.

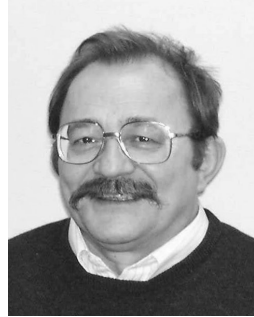

Mietek A. Brdys (1946-2015) received the MSc degree in electronic engineering and the $\mathrm{PhD}$ and DSc degrees in control systems from the Institute of Automatic Control at the Warsaw University of Technology in 1970, 1974 and 1980, respectively. In 1992 he became a full professor of control systems in Poland. During his career he was the head, a visiting professor, a staff member and a consultant at many universities, companies and organisations (IEEE and IFAC). His research was supported by the UK and Polish research councils, industry and the European Commission. He was the author and the co-author of about 220 refereed papers and six books. His research included intelligent decision support and control of large scale complex systems, robust monitoring and control, softly switched robustly feasible model predictive control and integrated monitoring, control and security of critical infrastructure systems. The applications included environmental systems, technological processes, power smart grids and defence systems.

Received: 13 June 2016

Revised: 9 December 2016

Re-revised: 25 February 2017

Accepted: 16 March 2017 